\title{
Constant Motion Delivery of Broadband Light Energy for Skin Tightening in Korean Patients
}

Sang Ju Lee ${ }^{1}$

Sung Bin $\mathrm{Cho}^{2,3}$

${ }^{1}$ Yonsei Star Skin \& Laser Clinic, Seoul, Korea

${ }^{2}$ Department of Dermatology and Cutaneous

Biology Research Center, International St. Mary's Hospital, Catholic Kwandong University College of Medicine, Incheon, Korea

${ }^{3}$ Kangskin Sillim Dermatology Clinic, Seoul, Korea
Received September 7, 2017

Revised September 18, 2017

Accepted September 19, 2017

\section{Correspondence}

Sung Bin Cho

Department of Dermatology and Cutaneous

Biology Research Center, International St. Mary's Hospital, Catholic Kwandong University College of Medicine, 25 Simgok-ro, Seo-gu, Incheon 22711, Korea

Tel.: +82-32-290-3141

Fax: +82-32-290-3142

E-mail: drsbchođagmail.com

(C) Korean Society for Laser Medicine and Surgery

(c) This is an open access article distributed under the terms of the Creative Commons Attribution NonCommercial License (http://creativecommons.org/ licenses/by-nc/4.0) which permits unrestricted noncommercial use, distribution, and reproduction in any medium, provided the original work is properly cited.
Broadband light (BBL) induces photothermolysis of key chromophores in the skin to achieve overall clinical improvement in aged skin. In the present case series, we describe three Korean patients who were treated with BBL using a constant motion delivery technique for skin tightening. The patients were treated with one or two sessions of BBL along the midface, lower face, and upper part of the neck using an 800ST cut-off filter, an energy intensity of $5 \mathrm{~W} / \mathrm{cm}^{2}$ to $10 \mathrm{~W} / \mathrm{cm}^{2}$, a pulse duration of 10 seconds, and a cooling crystal temperature of $25^{\circ} \mathrm{C}$ via a constant motion technique. The estimated total accumulation energy was approximately $15,000 \mathrm{~J} /$ session to $20,000 \mathrm{~J} /$ session. At one month after the final treatment, patients showed remarkable clinical improvement in overall skin texture and tone. Objective clinical assessment revealed marked improvement in zygomatic wrinkles, nasolabial folds, and perioral expression wrinkles at the cheek in the mid-face, as well as marionette lines and jaw lines in the lower face. Moreover, no major side effects were reported in our patients of Fitzpatrick skin phototype III or IV.

\section{Key words}

Broadband light; Polychromatic light; Skin tightening; Skin laxity; Constant motion technique 


\section{INTRODUCTION}

Broadband light (BBL) devices emit radiation of the broad electromagnetic spectrum via a flashlamp at the pulse duration of milliseconds. ${ }^{1-4}$ Photons of different wavelengths emitted by BBL devices can reach different depths of the skin and can be absorbed by various chromophores. ${ }^{2,3}$ Thereby, BBL systems have been effectively applied in the treatment of pigmented and vascular diseases, as well as in hair removal and skin rejuvenation, by regulating wavelengths using particular cut-off filters, fluences, and pulse durations and intervals. ${ }^{1,4}$ BBL-induced noninvasive skin rejuvenation or tightening facilitates clinical improvement of skin texture and rhytides, along with concomitant improvement in pigmented or vascular lesions. ${ }^{4-6}$

Technological advances in BBL devices have enabled clinics to irradiate qualified high-intensity light sources into the skin in rapid succession that has consistently been found to achieve satisfactory clinical improvements. However, optimized treatment settings for skin rejuvenation have not been fully elucidated in Asian patients. In the present case series, we describe three Korean patients who were treated with BBL using a constant motion delivery technique for skin tightening without major side effects.

\section{CASE REPORT}

Three Korean patients la 46-year-old male, a 29-yearold female, and a 32-year-old female) of Fitzpatrick skin phototype III-IV were scheduled to undergo constant motion delivery of BBL energy for skin tightening. All of the patients presented with no remarkable history of prior skin tightening or face lifting, such as systemic or topical retinoid therapy, skin resurfacing procedures (e.g., chemical or mechanical dermabrasion or laser resurfacing), fractional laser treatment (e.g., non-ablative or ablative),

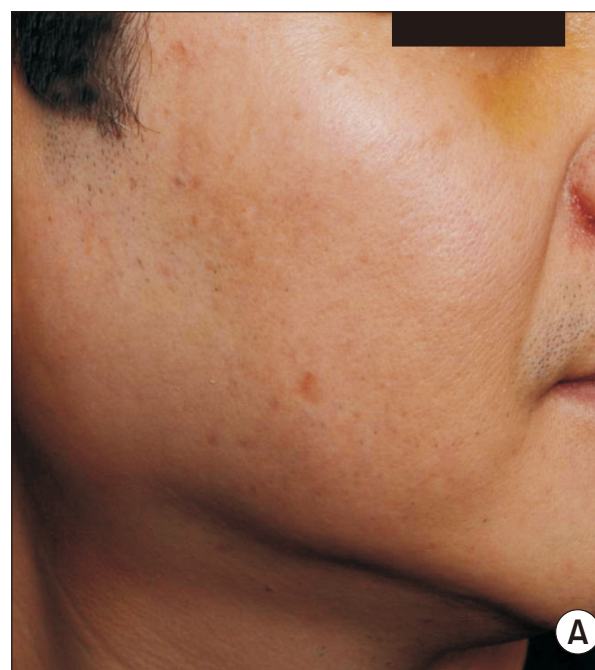

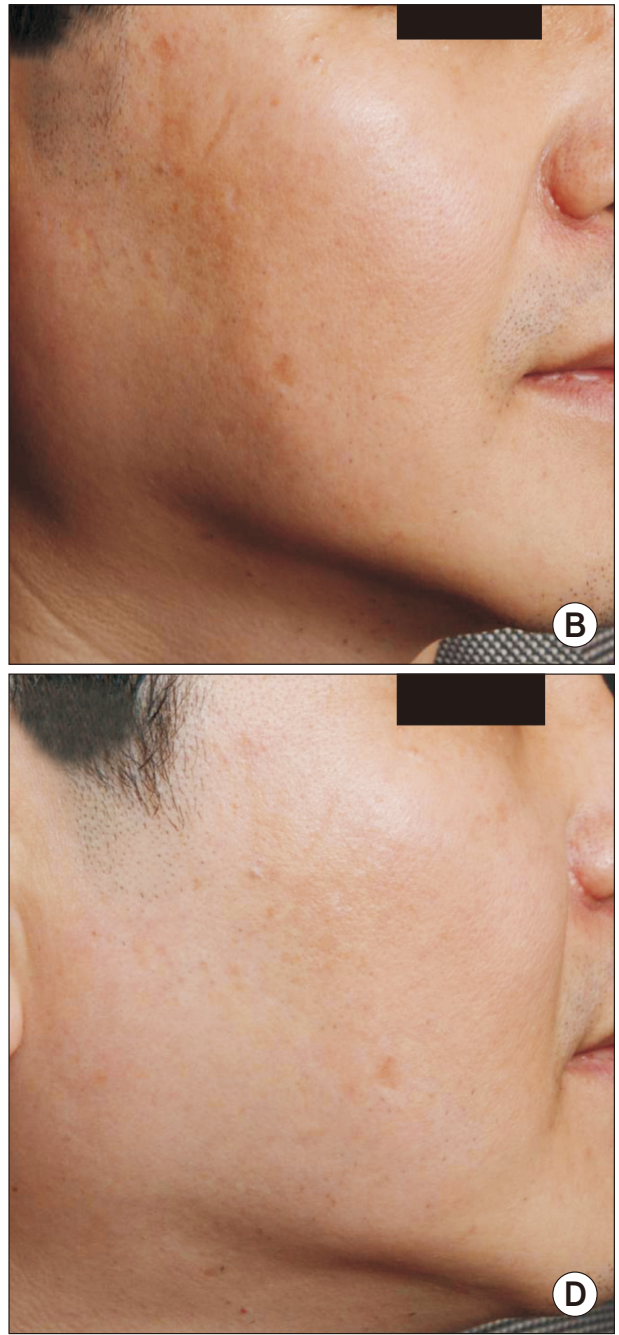

Fig. 1. Photographs of a 46-year-old male patient $(\mathrm{A}, \mathrm{C})$ at baseline and $(\mathrm{B}, \mathrm{D})$ at one month after two sessions of broadband light (BBL) treatment using a constant motion technique. Remarkable clinical improvement in zygomatic wrinkles, nasolabial folds, perioral expression wrinkles, marionette lines, and jaw lines is apparent. (A, B) Right oblique view, (C, D) right lateral view. 


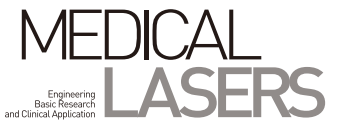

radiofrequency treatment (e.g., monopolar or bipolar; invasive or noninvasive), intense focused ultrasound treatment, botulinum toxin injection, thread implantation, filler or tissue activator injection, or face lift surgery within the last six months. Moreover, none of the three patients had a propensity for keloids or hypertrophic scars.

After obtaining written informed consent, a 46-yearold Korean male patient was treated with two sessions of BBL treatment using SkinTyte II ${ }^{\mathrm{TM}}$ (Sciton, Palo Alto, CA, USA) at 4-week intervals. Each treatment session began by gentle cleansing of the face and neck with a mild soap and $70 \%$ ethanol without topical or systemic anesthesia. Then, BBL therapy was performed on the mid-face, lower face, and upper part of the neck with an 800ST cut-off filter (Sciton), an intensity of $10 \mathrm{~W} / \mathrm{cm}^{2}$, a pulse duration of 10 seconds, and a cooling crystal temperature of $25^{\circ} \mathrm{C}$ via a constant motion technique.

The two Korean female patients were treated along the mid-face, lower face, and upper part of the neck with one session of BBL treatment using SkinTyte ${ }^{\mathrm{TM}}{ }^{\mathrm{M}}$ with the 800ST cut-off filter, an intensity of $5 \mathrm{~W} / \mathrm{cm}^{2}$, a pulse duration of 10 seconds, and a cooling crystal temperature of $25^{\circ} \mathrm{C}$ using a constant motion technique. During the BBL treatment, a Smoothie Adapter ${ }^{\top \mathrm{M}}$ (Sciton) was adapted to the $\mathrm{BBL}$ handpiece for covering the sides and edges of the crystal.

The estimated total accumulation energy was approximately $15,000 \mathrm{~J} /$ session to $20,000 \mathrm{~J} /$ session at the treatment endpoint of a skin surface temperature measuring $40^{\circ} \mathrm{C}-42^{\circ} \mathrm{C}$. The treated areas were cooled with icepacks. No prophylactic systemic corticosteroids or antibiotics were prescribed. The patients were recommended to apply broadband sunscreens and emollients, but not topical bleaching and retinoid agents, during the course of treatment and follow-up periods.

At one month after the final treatment, patients exhibited remarkable clinical improvement in overall skin texture, contour of the face, and skin tone (Fig. 1-3). Upon
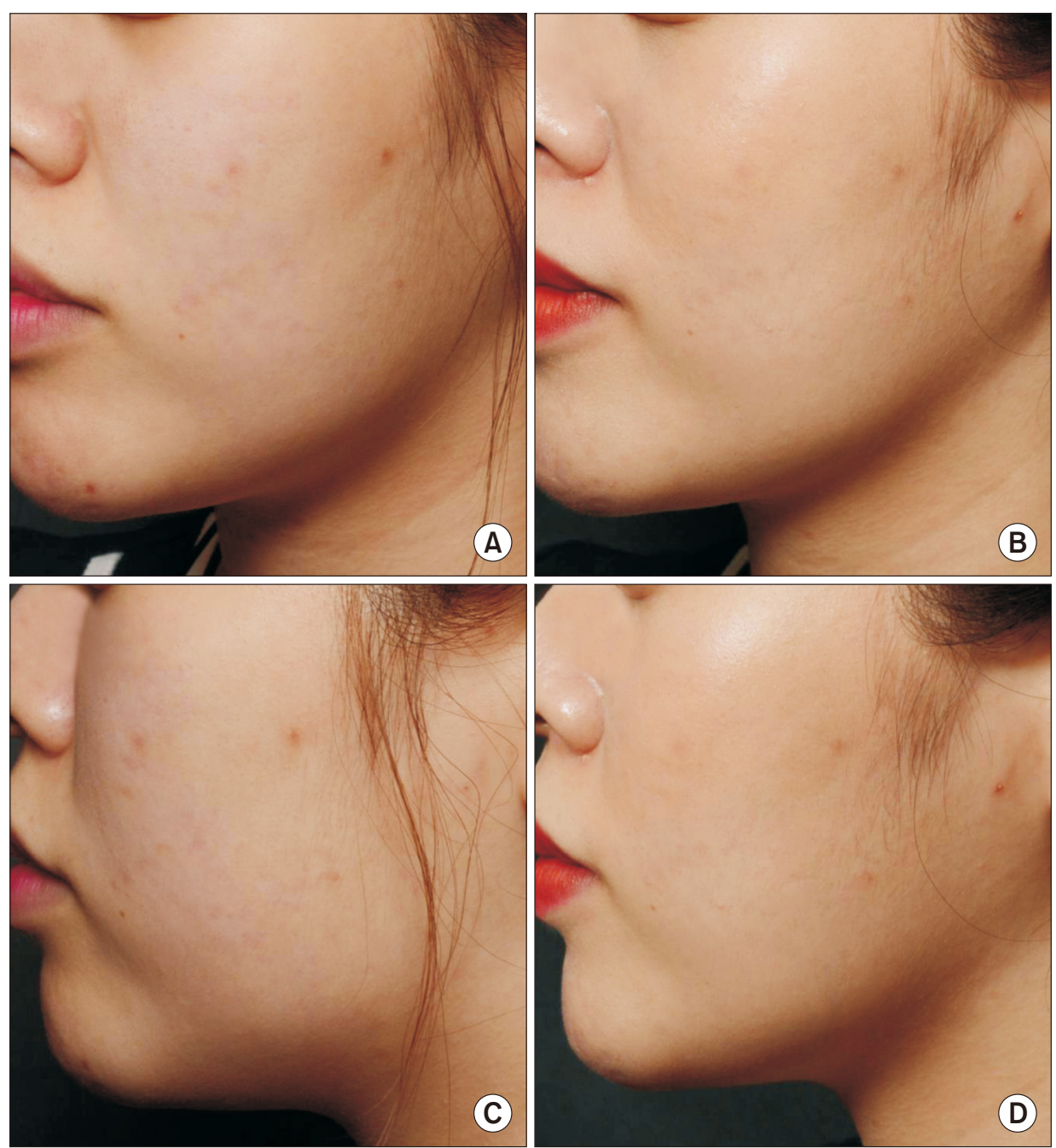

Fig. 2. Photographs of a 29-year old female patient $(\mathrm{A}, \mathrm{C})$ at baseline and $(\mathrm{B}, \mathrm{D})$ at one month after one session of BBL treatment using a constant motion technique. Noticeable clinical improvement in nasolabial folds, marionette lines, and jaw lines is apparent. (A, B) Left oblique view, $(\mathrm{C}, \mathrm{D})$ left lateral view. 
BBL for Skin Tightening Sang Ju Lee and Sung Bin Cho
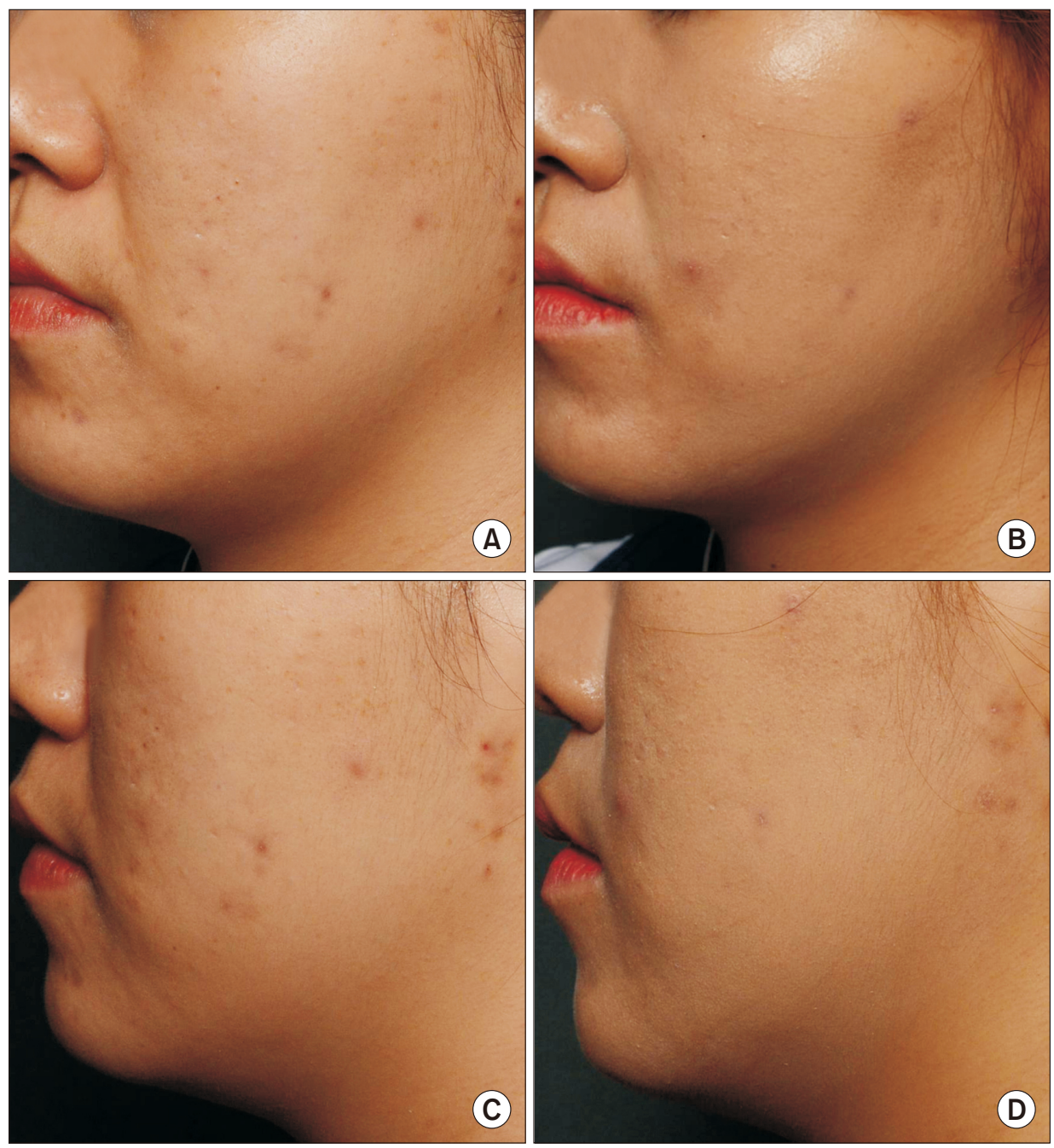

Fig. 3. Photographs of a 32-year-old female patient $(\mathrm{A}, \mathrm{C})$ at baseline and $(\mathrm{B}, \mathrm{D})$ at one month after one session of BBL treatment using a constant motion technique. Clinical improvement in nasolabial folds, marionette lines, and jaw lines is apparent. (A, B) Left oblique view, $(\mathrm{C}, \mathrm{D})$ left lateral view. objective clinical assessment, we noted marked improvement particularly in zygomatic wrinkles, nasolabial folds, and perioral expression wrinkles at the cheek in the midface, as well as marionette lines and jaw lines in the lower face. Furthermore, no major side effects, including oozing, scaling, crusting, burn, secondary skin infection, prolonged edema or erythema, post-therapy hyperpigmentation or hypopigmentation, and scarring, were encountered.

\section{DISCUSSION}

Skin aging, including photoaging and intrinsic aging, elicits the characteristic clinical features of wrinkles, coarse texture, and alterations of pigment and vascular components in the skin. ${ }^{5}$ To achieve overall clinical improvement in aging skin, the provocation of photobiologic reactions via the simultaneous photothermolysis of key chromophores is needed. To do so, a combination of laser devices at different wavelengths or the use of polychromatic high-intensity light sources has been chosen for treating aging skin.

Therapeutic modalities for recovering aging skin via the delivery of particular energy sources include carbon dioxide or erbium:yttrium aluminum garnet laser resurfacing, non-ablative or ablative fractional laser, monopolar or bipolar radiofrequency, and intense focused ultrasound treatments. The clinical efficacy of laser resurfacing or ablative fractional laser treatment for skin rejuvenation has been well accepted; however, significantly higher risks of major side effects, compared to the other modalities, limit the use thereof in Asian patients. ${ }^{7}$ Thus, noninvasive or non-ablative energy-delivering devices have been relied upon for skin tightening, allowing clinicians to generate zones of thermal coagulation in target skin layers while preserving the structural integrity of the skin. ${ }^{7}$

$\mathrm{BBL}$ treatment has been found to increase the activity of fibroblasts and the amounts of collagen type I and type 
III. ${ }^{8-10}$ The rearrangement of collagen and elastic fibers has also been reported for BBL irradiation. ${ }^{9.10}$ Moreover, the expression of age-related protein biomarkers, including advanced glycosylation end products, can be recovered by BBL treatment. ${ }^{8}$ The constant motion delivery technique, which was used in this report, was deemed to offer the advantages of better-balanced delivery of BBL energy to target tissue, a shorter procedure time, and a lower risk of side effects, compared to the static operation technique. Thereby, the non-invasive BBL treatment could be of use for skin tightening in the aged skin of Asian patients to induce fibroblast activation and neocollagenesis. Clinical improvement in skin tone and agingrelated alterations of pigment and vascular components could also be expected.

In conclusion, we treated three Korean patients using constant motion delivery of BBL energy using for skin tightening in the present case series. Our patients experienced noticeable clinical improvements in various clinical assessment parameters that could reflect the therapeutic efficacy of BBL treatment for skin tightening. Moreover, no major side effects, particularly burn, post-treatment erythema, and postinflammatory hypo- or hyperpigmentation, were reported in our patients of Fitzpatrick skin phototype III or IV. Notwithstanding, further prospective, controlled investigations are required to confirm our findings.

\section{ACKNOWLEDGEMENTS}

We would like to thank Duke Song (Consultant; Sciton, Palo Alto, CA, USAl for his assistance with technical support. We would also like to thank Anthony Thomas Mil- liken, ELS at Editing Synthase (https://editingsynthase. $\mathrm{com})$ for his help with the editing of this manuscript.

\section{REFERENCES}

1. Wat H, Wu DC, Rao J, Goldman MP. Application of intense pulsed light in the treatment of dermatologic disease: a systematic review. Dermatol Surg 2014;40:359-77.

2. Bjerring P. Clinical comparison of hair reduction using the newest generation IPL and a first generation ruby laser. J Cutan Laser Ther 2000;2:211.

3. Raulin C, Schroeter CA, Weiss RA, Keiner M, Werner S. Treatment of port-wine stains with a noncoherent pulsed light source: a retrospective study. Arch Dermatol 1999;135:679-83.

4. DiBernardo BE, Pozner JN. Intense pulsed light therapy for skin rejuvenation. Clin Plast Surg 2016;43:535-40.

5. Bitter PH. Noninvasive rejuvenation of photodamaged skin using serial, full-face intense pulsed light treatments. Dermatol Surg 2000;26:835-42; discussion 843.

6. Kligman DE, Zhen Y. Intense pulsed light treatment of photoaged facial skin. Dermatol Surg 2004;30:1085-90.

7. Park SH. Fractional laser; NAFL and AFL. Med Lasers 2015;4:1-9.

8. Shin JU, Lee WJ, Oh SH, Kim DY, Kim DS, Jung I, et al. Altered vimentin protein expression in human dermal microvascular endothelial cells after ultraviolet or intense pulsed light treatment. Lasers Surg Med 2014;46:431-8.

9. Babilas P, Schreml S, Szeimies RM, Landthaler M. Intense pulsed light (IPL): a review. Lasers Surg Med 2010;42:93-104.

10. Feng Y, Zhao J, Gold MH. Skin rejuvenation in Asian skin: the analysis of clinical effects and basic mechanisms of intense pulsed light. J Drugs Dermatol 2008;7:273-9. 